Digital Press Social Sciences and Humanities

La cohésion grammaticale en langue française au discours de la parole Emmanuel Macron

Khotibul Umam, Sri Rejeki Urip and Bernadus Wahyudi Joko Santoso

Proceeding of Conférence internationale sur le français 2018

Joesana Tjahjani, Merry Andriani, Sajarwa, Wening Udasmoro (eds) 


\title{
La cohésion grammaticale en langue française au discours de la parole Emmanuel Macron
}

\author{
Khotibul Umam*, Sri Rejeki Urip et Bernadus Wahyudi Joko Santoso \\ Faculté des Langues et des Arts, Universitas Negeri Semarang, Semarang, Indonesia \\ *e-mail: khotibulumam316@gmail.com
}

\section{Résumé}

La langue a une fonction idéationnelle qui signifie que la langue peut être utilisée comme un instrument pour exprimer des idées aux autres. Une des formes des idées qui peut être utilisées dans la langue est le discours de la parole. Le discours est l'unité de langue complète. En géneral, le discours est organisé par une structure claire. Pour former un propre discours, le discours doit avoir deux éléments essentiels, ce sont la cohésion et la cohérence. La cohésion est une rélationsemantique dans une paragraphe. Elle est partagée en deux parties, ce sont la cohésion lexicale est la cohésion grammaticale.Cette recherche a pour but de savoir la cohésion grammaticale dans le discours d'Emmanuel Macron. Selon Halliday et Hasan (1976, p. 1) le concept de cohésion est l'unité sémantique qui réfère aux relations de sens qui existent dans le texte, et qui le définit comme le texte. Selon Maingueneau, en général, on considère que la cohésion est le résultat de l'enchaînement les linéarité des propositions dans le texte, alors que la cohérence s'appuie sur la cohésion mais fait aussi intervenir des contraintes globales, non liniéaires, attachées en particulier au contexte, au genre de discours. La source des données sont les discours d' Emmanuel Macron, le Président de la République Française. Ces données sont téléchargées de Youtube en Canal de BFM TV. De cette recherché, on peut conclure que (1) Il y a quatre catégories de la cohésion grammaticale, ce sont la référence, la substitution, l'ellipse, et la conjonction (connecteur), et (2) La référence personnelle est la catégorie la plus dominante dans ce discours. La conclusion de cette recherche est (1) Toutes les quatre marques de la cohésion grammaticale apparaîssent dans les discours, (2) la cohésion qui ont été trouvée sont (1) 167 références, 4 substitutions, 20 ellipses, 45 conjonctions (connecteurs), et la cohésion la plus dominante est la référence personnelle exophore utilisant le pronom personnel 'nous'.

\section{Mots-clés}

cohésion grammaticale, discours, Emmanuel Macron

\begin{abstract}
The language has an ideational function, which means that the language can be used as a tool to express ideas to others. One of forms of ideas that can be used in the language is the speech discourse. In general, the speech is organized in a clear structure. A well-formed speech must have two essential elements, cohesion and coherence. Cohesion is a semantic relationship in the paragraph, which is divided into lexical cohesion and grammatical cohesion. This research aims to identify the grammatical cohesion in Emmanuel Macron's speech in order to analyze the possibility of using grammatical cohesion. This research uses both theoretical approach (the approach of discourse) and methodological (qualitative descriptive analytical) approach. The data collection is carried out by recording technique. The method of analysis uses the distributional method with BUL (Bagi Unsur Langsung) technique. The results are presented by using the method and the formal and informal technique. The analysis resulted in that (1) there are four categories of grammatical cohesion, i.e. reference, substitution, ellipse, and the conjunction (connector), and (2) the personal reference is the dominant category in the speech. The conclusion: the data obtained are 167 references, 4 substitutions, 20 ellipses, 45 conjunctions (connector), and the most dominant is the exosphoric personal reference with the personal pronoun 'we'.
\end{abstract}

\section{Keywords}

grammatical cohesion, speech, Emmanuel Macron 


\section{Introduction}

La communication est une nécessité de base pour tous les gens. La langue devient une partie importante de la communication parce qu'elle peut être utilisée pour transmettre des messages aux autres. La langue a une fonction qui signifie que la langue peut être utilisée comme un instrument pour exprimer des idées aux autres. L'une des formes des idées qui peut être utilisée dans la langue est le discours (Zaimar \& Harahap, 2009, p. 1).

Le discours est l'unité de langue complète. En général, le discours est organisé par une structure claire. Pour former un propre discours, le discours doit avoir deux éléments essentiels, ce sont la cohésion et la cohérence.

Le concept de cohésion est l'unité sémantique, cela réfère aux relations de sens qui existent dans le texte, et qui le définit comme le texte (Halliday \& Hassan 1976, p. 1). Selon Maingueneauu, en général, on considère que la cohésion est le résultat de l'enchaînement les linéarité des propositions dans le texte, alors que la cohérence s'appuie sur la cohésion mais fait aussi intervenir des contraintes globales, non liniéaires, attachées en particulier au contexte, au genre de discours (Maingueneau, 1996, p. 16).

La cohésion est divisée en deux parties, ce sont la cohésion lexicale et la cohésion grammaticale. La cohésion lexicale est une relation sémantique qui est exprimée par les éléments lexicaux. Alors que la cohésion grammaticale est une relation sémantique qui est exprimée par les éléments grammaticaux.

Cette recherche a pour but de savoir la cohésion grammaticale dans le discours de l'actuel président de la République Française. Les chercheurs veulent aussi connaître la catégorie de la cohésion la plus dominante dans les discours.

\section{Méthodologie de recherche}

Dans cette recherche, les chercheurs utilisent la théorie de deux linguistes. Ce sont la théorie de Cohésion de Hasan et Halliday (1976) et celle de Maingueneau. Les chercheur ont aussi observé quelques recherches precedents.. Ce que Urip $(2009$, p. 231) dit dans la séminaire linguistique litterature et civilisation françaises contemporaines "the verypopularscholars of discourseanalysis in Indonesia are Halliday and Hasan". Dans cet article, les cherecheurs essaient d'utiliser de point de vu pour analyser le discours par la théorie Halliday et Hasan et celle de Maingueneau.

Maingueneau dit qu'en général, on considère que la cohésion est le résultat de l'enchaînement des linéarité des propositions du texte. Selon Halliday et Hasan (1976, p. 1) le concept de cohésion est l'unité sémantique, cela se réfère aux relations de sens qui existent dans le texte, et qui le définit comme le texte. Halliday et Hassan divise la cohésion en deux catégories, ce sont la cohésion lexicale et la cohésion grammaticale qui a quatre catégories, ce sont références, substitutions, el lipses, et conjonctions.

\section{Discussion}

\subsection{Réference}

La référence est une partie de la cohésion grammaticale qui est indiquée par l'utilisation d'un mot ou groupe de mots qui se réfèrent à un autre mot ou à groupe de mots ou aux autres unités grammaticales. Il y a deux systèmes pour se référer, ce sont l'exophoreet l'endophore. L'exophore ou "référence situationnelle ", c'est la référence de l'expression qui se trouve dans l'espace non discursif. L'endophore recouvre les relations d'anaphore et cataphore, c'est à dire les divers phénomènes de reprise d'un segment par un autre dans un même ensemble textuelle. Ensuite, en parlant de phénomènes anaphores, on utilise généralement l'expression antécédente, mais en parlant d'anaphore, il est possible d'employer le terme "anaphorisé » pour référer et le terme " anaphorisant» pour la conctituant remplacé. En parlant de la cataphore, on peut utiliser le terme «cataphorisé » pour exprimer l'expression référentielle et le terme "cataphorisant» pour le contraire (Maingueneau, 1994, p. 139). La référence a trois catégories. Ce sont la référence personnelle, la référence démonstrative, et la référence comparative.On peut trouver ci-dessous les données de chaque référence et leurs analyses.

\subsubsection{Référence Personnelle Exophore}

(1) Tout le monde nous disait que c'était impossible, mais ils ne connaissaient pas la France. 
Le pronom personnel nous qui est trouvé dans cette phrase est la référence personnelle exophore. Ce pronom se réfère a une chose qui existe au dehors du texte. Le pronom personnnel nous se réfère à Emmanuel Macron (je) qui joue le rôle de destinateur et les citoyens français (ils) qui jouent le rôle de destinataires.

\subsubsection{Référence Démonstrative Exophore}

(2) Je sais nos désaccords, je les respecterai, mais je serai fidèle à cet engagement puis, je protègerai la République.

Adjectif démonstratif 'cet' dans cette phrase est la référence démonstrative exophore qui se réfère au sujet au dehors du texte. C'est l'engagement d'Emmanuel Macron au futur pour protéger la République parce qu'il est le Président qui a été élu par l'élection présidentielle.

\subsubsection{Référence Comparative Exophore}

(3) Ils attendent que nous portions une nouvelle espérance, un nouvel humanisme, celui d'un monde plus sûr, d'un monde de libertés défendues, d'un monde de croissance, de plus de justice, de plus d'écologie.

Dans cette phrase le mot 'plus' est la référence comparatif exophore. Le mot 'plus' se réfère au sujet qui existe au dehors du texte. C'est-à-dire que le constituant remplacé ne se trouve pas avant ou après de lui (Sajarwa, 2009, p. 184).. La phrase (3), veut dire qu'on connait que la France est un pays qui a la dévise : liberté, égalité, fraternité, dans ce cas là, la France doit devenir un pays qui a plus de justice qu'avant. 'Plus d'écologie' veut dire que la France est un pays qui concern etoujours à l'environement.

\subsubsection{Référence Personnelle Endophore Anaphore}

(4) Tout le monde nous disait que c'était impossible, maisils ne connaissaient pas la France!

Pronom personnel ils est la référence personnelle endophore anaphore. Le pronom personnel "Ils" se réfère au sujet qui est cité précédemment c'est à dire "tout le monde". Comme anaphorisant est le pronom personnel "ils" et comme anaphorisé est "tout le monde".

\subsubsection{Référence Démonstratif Endophore Anaphore}

(5)Peuple de France, ici, rassemblé au Louvre, nous avons la force, nous avonsl'énergie, nous avons la volonté, celle qui nous aportés, qui afait ce que nous sommes.

Dans le discours ci-dessus, pronom démonstratif celle est la référence démonstrative endophore anaphore. Celle se réfère au sujet qui est cité précédemment c'est à dire le mot "la volonté". Comme anaphorisant est le pronom démonstratif "celle" et comme anaphorisé est lemot précédemment, la volonté.

\subsubsection{Référence Démonstrative EndophoreCataphore}

(6) Et je veux enfin avoir un mot pour ceux qui ont voté aujourd'hui pourMadame LE PEN -ne les sifflez pas- ils ont exprimé aujourd'hui une colère, un désarroi, parfois des convictions.

Dans le discours au-dessus, pronom démonstratif ceux est la référence démonstratif endophore cataphore. Ceux se réfère au sujet qui est citéaprès, c'est-à-dire ont voté aujourd'hui pour Madame LE PEN. Comme cataphorisant est ceux et comme cataphorisé est voté aujourd'hui pour Madame LE PEN.

\subsection{Subtitution}

La substitution est une partie de la cohésion grammaticale indiquée par le changement des éléments dans une texte avec des autres éléments. Hassan et Halliday (1976 : 90) divisent la substitution en trois catégories, ce sont la substitution nominale, la substitution verbale, et enfin la substitution clausale.

\subsubsection{Substitution Nominale}

(7) Je sais nos désaccords, je les respecterai, mais je serai fidèle à cet engagement pris : je protègerai la République.

Pronom 'les' est COD 'Complément Objet Direct', ce pronom substitue au sujet précédent, 'nosdésaccords'. On peut observer dans laphrase suivante.

(7a)Je sais nos désaccords, je les respecterai, mais je serai fidèle à cet engagement pris : je protègerai la République.

(7b) Je sais nos désaccords, je respecterai nos désaccords, mais je serai fidèle à cet engagement pris : je protègerai la République. 
La phrase (7b) est acceptée comme la substitution 'nos désaccord' dans la phrase (7a). En voyant la forme acceptée et grammaticale, le chercheurpeut conclure que l'élément substituant et l'élément substitué sont dans la même classe et le même rôle. Pronom 'les' est une unité grammaticale utilisée pour substituer 'nos désaccords'.

\subsubsection{Substitution Clausal}

(8) Merci de votre confiance, merci du temps que vous avez donné, merci de votre engagement à toutes et tous, merci des risques pris parcertains, je les sais.

Pronom 'les' est COD 'Complément Objet Direct', ce pronom substitue au sujet précédent 'des risques pris par certains'. On peutobserverdanslaphrasesuivante.

(8a) Merci de votre confiance, merci du temps que vous avez donné, merci de votre engagement à toutes et tous, merci des risques pris par certains, je les sais.

(8b) Merci de votre confiance, merci du temps que vous avez donné, merci de votre engagement à toutes et tous, merci des risques pris par certains, je sais des risques pris par certains.

La phrase (8b) est acceptée comme la substitution 'des risques par certains' dans la phrase (8a). En voyant la forme acceptée et grammaticale, le chercheur peut conclure que l'élément substituant et l'élément substitué sont dans la même classe et le même rôle. Pronom 'les' est une unité grammaticale utilisée pour substituer 'des risques par certains'.

\subsection{Ellipse}

Selon Maingueneau et Haroche (1983: 144), L'ellipse supprime les mots nécessaire à la construction de la phrase pour la rendre pleine et entière, mais inutiles au sens, parce que ceux qui sont énoncés les font aisément suppléer. Halliday et Hassan divisent l'ellipse en trois catégories, ce sont l'ellipse nominale, l'ellipse verbale, et l'ellipse clausale.

\subsubsection{Ellipse Nominal}

(9) Notre tâche est immense, mes amis, et elle exigera l'engagement de chacun : $\emptyset$ de nos armées, $\emptyset$ de nos forces de l'ordre, $\emptyset$ de tous nos services publics, votre engagement !

L'ellipse est marquée ave c $\emptyset$. Dans cette phrase, il y a le nom qui n'est pas mentionné, ce nom est 'l'engagement'. Si on le réécrit complètement, elle devient :

(9a) Notre tâche est immense, mes amis, et elle exigera l'engagement de chacun : l'engagement de nos armées, l'engagement de nos forces de l'ordre, l'engagement de tous nos services publics, votre engagement!

\subsubsection{Ellipse Verbal}

(10) Nous ne céderons rien à la peur, $\emptyset$ à la division, $\emptyset$ au mensonge, $\emptyset$ à l'ironie, $\emptyset$ à l'entre-soi, $\emptyset$ à l'amour du déclin ou de la défaite.

L'ellipse est marquée avec $\emptyset$. Dans cette phrase, il y a le verbe qui n'est pas mentionné, ce verbe est 'cederer'. Si on le réécrit complètement, elle devient:

(10a) Nous ne céderons rien à la peur, nous ne céderons rien à la division, nous ne céderons rien au mensonge, nous ne céderons même rien à l'ironie, nous ne céderons même rien à l'entre-soi, nous ne céderons même rien à l'amour du déclin ou de la défaite.

\subsubsection{Ellipse Clausal}

11) Et je veux enfin avoir un mot pour ceux qui ont voté aujourd'hui pour Madame LE PEN - ne les sifflez pas, ils ont exprimé aujourd'hui une colère, $\emptyset$ un désarroi, $\emptyset$ parfois des convictions.

L'ellipseestmarquéeavec Ø. Dans cette phrase, il y a l'élémentquin'est pasmentionné, c'est à dire 'ils ont exprimé aujourd'hui'. Si on le réécritcomplètement, elledevient :

(11a) Et je veux enfin avoir un mot pour ceux qui ont voté aujourd'hui pour Madame LE PEN - ne les sifflez pas, ils ont exprimé aujourd'hui une colère, ils ont exprimé aujourd'hui un désarroi, ils ont exprimé aujourd'hui parfois des convictions.

\subsection{Conjonction (Connecteur des phrases)}

Halliday et Hassan classifient la conjonction enquatrecatégories, ce sont la conjonction addition, la conjonction adversative, la conjonction causale, et enfin la conjonction temporel. Maingueneau avec la 
même concept classifie que la conjonction est des connecteurs entre phrases qui divisent quatre catégories. Ce sont connecteur d'addition, connecteur d'opposition, connecteur de cause/ conséquence, et connecteur de temps.

\subsubsection{Connecteur Additif}

(12)Merci, merci d'être là, de vous être battus avec courage et bienveillance pendant tant de mois.

Après avoir lu des marques linguistiques, le chercheur peut trouver un connecteur d'additif, c'est à dire le mot 'et'. Le connecteur 'et' a une fonction addition.

(12a) Merci, merci d'être là, de vous être battus avec courage pendant tant de mois.

(12b) Merci, merci d'être là, de vous être battus avec bienveillance pendant tant de mois.

(12c) Merci, merci d'être là, de vous être battus avec courage et bienveillance pendant tant de mois.

La phrase (12c) est la phrase (12a) et la phrase (12b) qui sont liées par le connecteur 'et'. Le connecteur 'et' est une unité grammaticale utilisée pour unir deux noms ,'courage' et 'bienveillance'

\subsubsection{Connecteur Opposition}

(13) Tout le monde nous disait que c'était impossible, mais ils neconnaissaient pas la France!

Après avoir lu des marques linguistiques, le chercheurpeut trouver un connecteur d'opposition, c'est à dire le mot 'mais'. Le connecteur 'mais' a une fonction d'opposer.

(13a) Tout le monde nous disait que c'était impossible.

(13b) Ils ne connaissaient pas la France.

(13c)Tout le monde nous disait c'était impossible, mais ils ne connaissaient pas la France

La phrase (13c) est le résultat de la combination de la phrase (13a) et la phrase (13b) qui sont liées par le connecteur 'mais'. Le connecteur 'mais' est une unité grammaticale utilisée pour unir deux proposition 'tout le monde nous disait que c'était impossible' et 'Ils ne connaissaient pas la France'.

\subsubsection{Connecteur de Cause et Conséquence}

(14) Ce lieu, c'est celui de la France, que le monde regarde, car ce soir, c'est l'Europe, c'est le monde qui nous regarde!

Après avoir lu des marques linguistiques, le chercheur peut trouver un connecteur de cause, c'est à dire le mot 'car'. Le connecteur 'car' a une fonction de causative.

(14a) Ce lieu, c'est celui de la France, que le monde regarde

(14b) Ce soir, c'est l'Europe, c'est le monde qui nous regarde !

(14c) Ce lieu, c'est celui de la France, que le monde regarde, car ce soir, c'est l'Europe, c'est le monde qui nous regarde!

La phrase (14c) est la combination de la phrase (14a) et la phrase (14b) qui sont liées par le connecteur 'car'. Le connecteur 'car' est une unité grammaticale utilisée pour unir deux proposition 'ce lieu, c'est celui de la France, que le monde regarde' et 'ce soir, c'est l'Europe, c'est le monde qui nous regarde !'.

\section{Conclusion}

Les suivants sont les conclusions de la recherche :

a. Il ya 4 catégories de la cohésion grammaticale danslasourcededonées.

b. De l'analyse des données, les chercheurs ont trouvé 167 références, 4 substitutions, 20 l'ellipses, et

45 connecteurs. La plupart de discours utilise la catégorie de la référence qui est la plus dominante, en particulier la référence personnelle et surtout le pronom personnel premier singulier.

En pourcentage, on peut conclure que dans la catégorie de référence, il y a 58,1\% références personnelles; $11 \%$ références démonstratives, et $1,7 \%$ références comparatives. Dans la catégorie de substitution, il y a 0,8 substitution nominale et clausale. Ensuite, dans la catégorie d'ellipse, il y a 3\% ellipses nominales, $4,3 \%$ ellipses verbales, et $0,8 \%$ ellipses clausales. Enfin, pour des connecteurs des phrases, Il y a $14,8 \%$ connecteurs additifs, $1,7 \%$ connecteurs d'opposition, et $3 \%$ connecteurs de cause et conséquence. 


\section{Références}

Halliday, M. A. K., \& Hasan, R. (1976). Cohesion in English. London: Longman.

Sajarwa. (2009). Définiet Indéfini : L'analyse Pragmatique de Nom Français (Séminaire Littérature,

Linguistique et Civilisation Françaises Contemporaines). Yogyakarta.

Urip, S. R. (2009). An Anlysis of Text of a Reader Comments From Lemonde.fr Using Schiffrin's Discourse Perspective (Séminaire Littérature, Linguistique et Civilisation Françaises Contemporaines).

Yogyakarta.

Zaimar, O. K. S., \& Harahap, A. B. (2009). Telaah Wacana. Jakarta: The Intercultural Institute. 